\title{
A REMARK CONCERNING LITTLEWOOD'S TAUBERIAN THEOREM
}

H. S. SHAPIRO

Let $\left\{a_{n}\right\}, n=0,1, \cdots$, be real numbers such that the series $\sum_{0}^{\infty} a_{n}$ is Abel summable to $s$. Then, by a well-known theorem of Littlewood, if

$$
a_{n}=O\left(\frac{1}{n}\right)
$$

the series converges to $s$. Professor Carleson has suggested to the author the question of whether the condition

$$
f(x)=\sum_{n=0}^{\infty} a_{n} x^{n} \text { is of bounded variation on }[0,1)
$$

(which plainly implies that $\sum a_{n}$ is Abel summable), implies the convergence of $\sum a_{n}$ under a substantially weaker Tauberian condition than (1). (Such a theorem would have useful applications in the theory of Fourier series.) We show, however, the following negative result:

THEOREM. Given any $\epsilon>0$, there is a sequence $a_{n}$ satisfying (2) with

$$
a_{n}=O\left(\frac{1}{n^{1-\epsilon}}\right)
$$

for which $\sum a_{n}$ is divergent.

Proof. We take $a_{0}=0, a_{n}=n^{-\alpha} \cos n^{\beta}$ for $n=1,2, \cdots$, where $\beta=1 / 2 k$ ( $k$ a positive integer) and $\alpha=1-\beta$. Note first that the series $\sum a_{n}$ diverges because the sum of consecutive terms in a block of terms having like sign does not tend to zero, as is readily verified. Hence the theorem will be proved if for every $k$ the function

$$
F(y)=\sum_{n=1}^{\infty} n^{-\alpha} \cos n^{\beta} e^{-n y}
$$

is of bounded variation (B. V.) on $[0, \infty)$. Setting

$$
G(t, y)=t^{-\alpha} \cos t^{\beta} e^{-t y},
$$

we have

Received by the editors November 7, 1963. 


$$
\begin{aligned}
F(y) & =\sum_{n=1}^{\infty} G(n, y)=\int_{1-0}^{\infty} G(t, y) d[t]=-\int_{1}^{\infty}[t] \frac{\partial G(t, y)}{\partial t} d t \\
& =G(1, y)+\int_{1}^{\infty} G(t, y) d t+\int_{1}^{\infty}(t-[t]) \frac{\partial G(t, y)}{\partial t} d t
\end{aligned}
$$

and we show that each of the three terms on the right is $\mathrm{B}$. V.

(i) $G(1, y)=e^{-y} \in \mathrm{B}$. V.

(ii) Consider next the last term on the right. Call it $H(y)$. Then

$$
\begin{aligned}
H^{\prime}(y) & =\int_{1}^{\infty}(t-[t]) \frac{\partial^{2} G(t, y)}{\partial t \partial y} d t, \\
\int_{0}^{\infty}\left|H^{\prime}(y)\right| d y \leqq & \int_{0}^{\infty} \int_{1}^{\infty}\left|\frac{\partial^{2} G(t, y)}{\partial t \partial y}\right| d t d y .
\end{aligned}
$$

From the estimate $\left|\partial^{2} G(t, y) / \partial t \partial y\right| \leqq\left(2 \beta t^{2 \beta-1}+y t^{\beta}\right) e^{-t y}(t \geqq 1)$, the finiteness of the double integral follows, and so $H \in \mathrm{B}$. V.

(iii) We are left finally with $\int_{1}^{\infty} G(t, y) d t$, and it suffices to study instead $\int_{0}^{\infty} G(t, y) d t$, since

$$
\int_{0}^{\infty}\left|\frac{\partial}{\partial y} \int_{0}^{1} G(t, y) d t\right| d y \leqq \int_{0}^{\infty} \int_{0}^{1} t^{1-\alpha} e^{-t y} d t d y<\infty .
$$

Now, $\int_{0}^{\infty} G(t, y) d t=\int_{0}^{\infty} t^{-\alpha} \cos t^{\beta} e^{-t y} d t=2 k z \int_{0}^{\infty} e^{-u^{2 k}} \cos z u d u$, where we have set $u=(t y)^{\beta}, z=y^{-\beta}$, and $\beta^{-1}=2 k$. It is enough to verify that the last integral represents a function of $\mathrm{B}$. $\mathrm{V}$. for $0 \leqq z<\infty$, and for this it suffices to remark that its derivative is of class $L^{1}$, since, in fact, both $\int_{0}^{\infty} e^{-u^{2 k}} \cos z u d u$ and $\int_{0}^{\infty} u e^{-u^{2 k}} \sin z u d u$ fall off at $\infty$ faster than any power of $z^{-1}$, being Fourier transforms of functions on $(-\infty, \infty)$ possessing $L^{1}$ derivatives of every order. The theorem is proved.

REMARK. The divergence of the above series, as well as the fact that it is Abel summable, follows from [1, Theorem 84], which implies that for any $k>-1, \sum_{1}^{\infty} n^{-\alpha} e^{i n^{\beta}}$ is summable $(C, k)$ if and only if $(k+1) \beta+\alpha>1$.

\section{BIBLIOGRAPHY}

1. G. H. Hardy, Divergent series, Clarendon Press, Oxford, 1949.

\section{New York UNIVERSITY AND} UNIVERSITY OF MICHIGAN 